\title{
ANÁLISE DA VIABILIDADE ECONÔMICA DE UMA SPIN-OFF DE ELECTROSPINNING NO BRASIL
}

\section{ECONOMIC FEASIBILITY ANALYSIS OF AN ELECTROSPINNING SPIN- OFF IN BRAZIL}

\author{
Ariadne Helena Pequeno de Oliveira ${ }^{1,2}$; Luciano Pisanu ${ }^{1,3}$; Helinando Pequeno de Oliveira ${ }^{2}$; Marcio \\ Luis Ferreira Nascimento ${ }^{1}$ \\ ${ }^{1}$ Universidade Federal da Bahia (UFBA), Salvador - BA - Brasil \\ ariadne.helena@ufba.br; mlfn@ufba.br \\ ${ }^{2}$ Universidade Federal do Vale do São Francisco (UNIVASF), Juazeiro - BA - Brasil \\ helinando.oliveira@univasf.edu.br \\ ${ }^{3}$ SENAI Cimatec, Salvador-BA-Brasil \\ luciano.pisanu@fieb.org.br
}

\begin{abstract}
Resumo
A produção de nanofibras a partir da técnica de electrospinning representa uma linha de atuação estratégica nacional tendo em vista os diversos tipos de materiais que podem ser obtidos, assim como um amplo leque de aplicações. Nanocompósitos poliméricos, constituídos geralmente pela junção de um polímero com substâncias inorgânicas torna possível a obtenção de novos materiais com características únicas e otimizadas quando comparadas às substâncias em separado. $O$ uso da técnica de eletrofiação (electrospinning) faz com que a interação entre os dois materiais na formação das fibras ocorra de maneira mais efetiva. Com o objetivo de melhor utilização dessa técnica, nesse trabalho foi analisada financeiramente a viabilidade de compra e instalação de um spin-off acadêmico visando a fabricação de nanofios pela técnica de electrospinning por uma incubadora de inovação de alta base tecnológica, vinculada a um laboratório de pesquisa em materiais brasileiro. A partir da utilização dos métodos de análise econômica de Valor Presente Líquido e Taxa Interna de Retorno foi possível verificar o potencial de lucratividade que a aquisição e operação de uma máquina de electrospinning pode fornecer para uma spin-off, levando em consideração tanto o aparato inicial necessário quanto as particularidades e variações apresentadas pelo mercado em que o produto está inserido.
\end{abstract}

Palavras-chave: fibra polimérica; electrospinning; spin-off; viabilidade econômica

\section{Introdução}

O desenvolvimento de nanofibras a partir da técnica de electrospinning (ou eletrofiação) tem sido um dos procedimentos mais utilizados em pesquisas científicas e produção industrial (NASCIMENTO et al., 2015). Diversos são os tipos de fibras que podem ser sintetizados e as aplicações que podem ser obtidas com esses materiais atingindo a escala nanométrica. Um exemplo bem ilustrativo encontra-se na formação de nanocompósitos poliméricos, que geralmente são 
constituídos de um polímero e uma substância inorgânica em menor quantidade (COSTA et al., 2015; ARAÚJO, NASCIMENTO, de OLIVEIRA, 2013; 2016).

A interação entre esses dois materiais pode ser otimizada à medida que temos uma melhor dispersão da substância inorgânica no polímero, o que pode ser obtido a partir da formação de nanofibras utilizando ao menos dois materiais formando um nanocompósito. A aplicação da técnica de electrospinning possibilita melhoras substanciais nas propriedades físico-químicas do material como: i) aumento da área superficial; ii) aumento da biodegradabilidade; iii) melhorias nas propriedades de barreira a diversos gases, podendo ser utilizados como biosensores e outros dispositivos (NASCIMENTO et al., 2015).

Assim, neste projeto foi analisada financeiramente a viabilidade de compra e instalação de um "spin-off" acadêmico (SOA) visando a fabricação de nanofios pela técnica de electrospinning por uma incubadora de inovação vinculada a um laboratório de pesquisa em materiais. Um "spin-off" acadêmico consiste numa empresa criada para explorar uma propriedade intelectual gerada a partir de um trabalho de pesquisa desenvolvido em uma instituição acadêmica (SCOTT SHANE apud LAGO et al, 2005).

A interação entre a comunidade acadêmica e as empresas é um assunto que tem despertado atenção tanto do ponto de vista teórico quanto do empírico, uma vez que, na economia do conhecimento, a ciência exerce uma forte influência na capacidade de inovação das empresas. A partir de métodos de Valor Presente Líquido e Taxa Interna de Retorno foi possível verificar o potencial de lucratividade que a aquisição e operação da máquina de electrospinning pode fornecer para a spin-off, levando em consideração todo o aparato inicial que deve ser providenciado pelo laboratório assim como as particularidades e variações apresentadas pelo mercado em que o produto está inserido, caracterizando uma verdadeira transferência de tecnologia (DIAS \& PORTO, 2013).

É de extrema importância a importação dessa tecnologia para o país, visto que em muitos laboratórios são utilizados aparatos simples que fornecem material sem grande controle de qualidade, confiabilidade, segurança ao processo de produção das nanofibras, e alta produtividade todos essas vantagens podem ser obtidas a partir da implantação spin-off em questão nesse projeto.

Antes de partir para a análise econômica do projeto será feita uma breve introdução à técnica, assim como serão citadas as características principais da máquina de electrospinning a ser utilizada na spin-off.

\section{A Técnica de Electrospinning}

O uso da técnica de electrospinning permite obter, de modo eficiente, fibras com diâmetro na escala de nanômetros (NASCIMENTO et al., 2015). Consiste em um método que envolve um processo eletrostático através da aplicação de uma alta tensão e baixa corrente, que torna possível a 
formação de fibras sólidas a partir de uma solução polimérica relativamente pouco viscosa através de forças eletrostáticas e de arraste (FRENOT e CHRONAKIS, 2003; ARAÚJO, NASCIMENTO, de OLIVEIRA, 2013; 2016).

Em 1934 foi depositada a primeira patente sobre a técnica de eletrofiação para a produção de microfibras por Anton Formhals (FORMHALS, 1934: United States Patent 1,975,504), e desde então esta se tornou uma área de bastante interesse, podendo ser aplicada a uma grande quantidade de materiais.

A obtenção dos fios pode ser realizada através da utilização de campos de alta tensão que variam entre $5 \mathrm{kV}$ a $50 \mathrm{Kv}$, e uma baixa corrente, entre 0,5 a $1 \AA$. Este processo envolve a aceleração de um jato do material fluido e o seu estiramento através da ação de um campo elétrico, que produz fibras com diâmetro entre 40 a 2000 nm (ALVES et. al., 2006; ARAÚJO, NASCIMENTO, de OLIVEIRA, 2016).

Primeiramente, é aplicada uma diferença de potencial na agulha ligada a uma seringa contendo a solução, formando uma gota em sua ponta. Quando a tensão elétrica aumenta e o campo elétrico é intenso o suficiente para vencer a tensão superficial da gota, esta fica altamente eletrificada. A superfície da gota alonga-se em forma cônica devido à diferença de potencial aplicada e realiza uma trajetória em espiral formando um cone, chamado Cone de Taylor, até alcançar o anteparo, como pode ser visto na Figura 1 (PORTELA, 2010; GRAFE, 2003; ARAÚJO, NASCIMENTO, de OLIVEIRA, 2013; 2013, 2016).

Figura 1 - Esquema ilustrativo da técnica de eletrofiação.

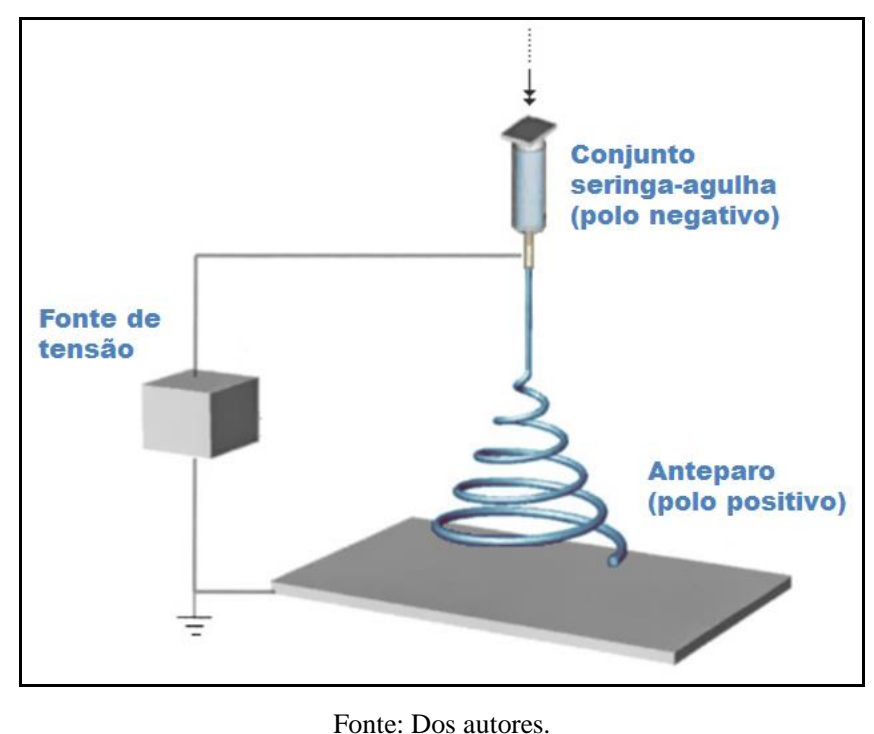

Segundo Guerrini (2006) e Nascimento et al. (2016), quando o jato é lançado o líquido evapora-se e o material solidifica-se formando assim nanofios, que quando agrupados formam uma espécie de tecido fibroso. A dimensão nanométrica das fibras sintetizadas faz com que elas possam ter diversas aplicabilidades, tais como: nanofiltros na indústria de separação, revestimento e 
proteção da pele humana, membranas multifuncionais, nanosensores, sistemas de liberação de fármacos, máquinas nano-eletrônicas, entre outros. (CONG, et. al., COSTA, et. al. , 2012; 2013; 2015)

De acordo com Oliveira (2012) e Nascimento et al. (2016), esse processo de eletrofiação pode ser afetado por diversas variáveis, como: concentração e viscosidade da solução polimérica (parâmetro de fundamental importância), tensão elétrica aplicada, presença de sais na solução, distância entre a extremidade da agulha e o coletor dos fios, diâmetro da agulha, entre outros. Por todos esses motivos faz-se necessário, para uma pesquisa científica de qualidade, a utilização de um equipamento desenvolvido industrialmente e exclusivamente para este fim, proporcionando a fabricação de nanofios para as mais diversas finalidades de forma rápida, altamente produtiva, confiável e com qualidade. Na próxima seção, são citadas algumas particularidades da máquina de electrospinning, cuja aquisição está sendo analisada neste projeto.

\subsection{Características da Máquina de Electrospinning}

A máquina a ser adquirida para a fabricação dos nanofios na spin-off é denominada NS LAB (Figura 2), fabricada pela ELMARCO (www.elmarco.com).

Algumas especificações da máquina são bastante interessantes, tanto para aplicações na indústria quanto na pesquisa científica, e algumas delas são importantes para a análise de viabilidade deste projeto. As características básicas do equipamento de eletrofiação são:

- Necessidade de controle de umidade e temperatura;

- Velocidade do substrato - 0 a $500 \mathrm{~mm} / \mathrm{min}$;

- Consumo de energia maior que $300 \mathrm{~W}$;

- Dimensões: largura de $1.193 \mathrm{~mm}$, comprimento de $820 \mathrm{~mm}$, altura de $1.276 \mathrm{~mm}$ e peso 195 $\mathrm{kg}$;

- Necessita de um operador em 3m x 3m de espaço;

- Recomenda-se ventilação de exaustão;

As principais características do processo são:

- O rendimento depende do polímero e do diâmetro da fibra;

- $\quad$ Temperatura de trabalho entre $20^{\circ} \mathrm{C}$ a $30^{\circ} \mathrm{C}$;

- Ambiente de trabalho com 20 a $40 \%$ de umidade relativa do ar;

- Tempo de aquecimento da máquina no mínimo em 30 minutos;

- Manutenção mensal;

- Produção de fibras a partir de polímeros solúveis ou não em água; 
- O diâmetro das fibras geradas é controlável entre $80 \mathrm{~nm}$ e $700 \mathrm{~nm}$.

Figura 2 - Máquina de electrospinning NS LAB e visão amplificada da produção dos fios.

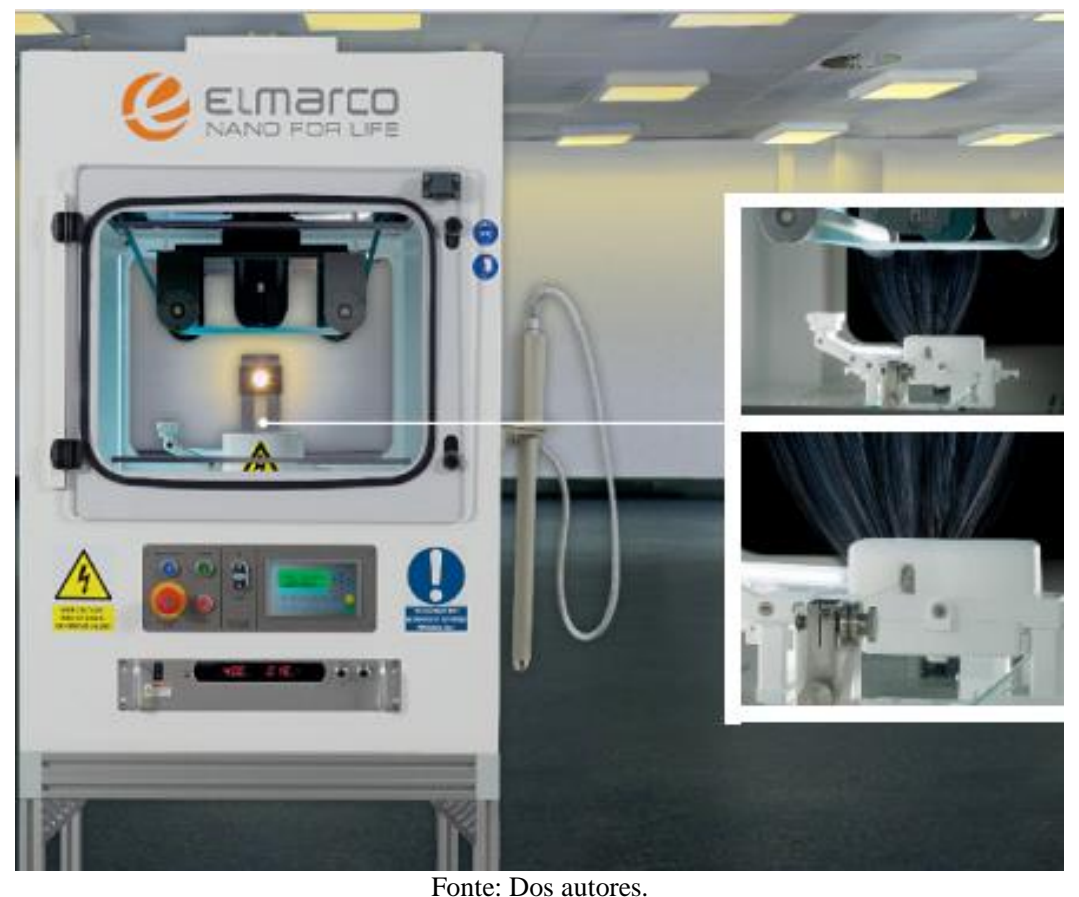

\section{Metodologia e Análise de Viabilidade do Projeto}

Nessa seção apresentamos a metodologia utilizada para análise de viabilidade econômica do projeto.

\subsection{Metodologia}

Segundo Gitman (2010), uma empresa despende capital tendo em vista um investimento, por diversas razões, seja ele para modernização, aumento da capacidade produtiva, sempre visando um retorno favorável em longo prazo. Um das características mais importantes a serem levadas em conta em uma decisão de um investimento diz respeito ao dimensionamento dos fluxos de caixa previstos a acontecerem ao longo dos anos em análise. Tendo em mãos esse fluxo de caixa por um determinado período de tempo podem ser utilizados alguns métodos de análise de investimentos, como é o caso do Valor Presente Líquido (VPL) e da Taxa Interna de Retorno (TIR), que serão utilizados nesse projeto visando a aquisição da máquina de electrospinnig.

De acordo com Tabosa et al. (2014), o método do VPL baseia-se na transferência, para o presente, dos fluxos de caixa esperados para um determinado projeto, descontados com relação à uma taxa de juros. Resumindo, ele pode ser definido como a soma algébrica dos valores descontados do fluxo de caixa associado ao mesmo. Podemos expressar a fórmula algébrica do VPL como: 


$$
V P L=-I+\sum_{t=1}^{n} \frac{F C t}{(1+j)^{t}}
$$

onde: $I=$ Investimento de Capital na data zero;

$n=$ Prazo de análise do projeto;

$F C t=$ Fluxo de caixa livre na data $t$;

$j=$ taxa mínima de atratividade.

Como critério de decisão para o método de análise por VPL, relaciona-se ao valor do mesmo se este valor for maior que zero então o projeto é financeiramente viável.

Segundo Samanez (2009), o método da Taxa Interna de Retorno (TIR), por sua vez, objetiva encontrar a taxa intrínseca de investimento. Essa taxa é aquela que, hipoteticamente, anularia o VPL a partir da seguinte relação:

$$
V P L=-I+\sum_{t=1}^{n} \frac{F C t}{(1+i *)^{t}}=0
$$

Sendo $i$ a taxa interna de retorno obtida pelo uso da equação acima, temos que se $i$ for maior que $j$ então o projeto é viável, pois a taxa interna de retorno ( $i$ ) é maior que a Taxa Mínima de Atratividade (ou TMA, indicado por $j$ ), que é a mínima taxa que o investidor se propõe a ganhar com o investimento.

\subsection{Análise Financeira}

A seguir será realizada a análise financeira do projeto a partir das técnicas de VPL e TIR.

\subsubsection{Investimento inicial}

A compra e instalação do equipamento, assim como outros recursos necessários para o funcionamento da spin-off acarretam alguns custos associados, como aquisição de um controlador de umidade, exaustor, reforma da sala onde será instalado o equipamento, custo de treinamento do operador e de instalação da máquina, assim como cadeiras e ar condicionado para a sala. Dessa forma, temos um investimento inicial de R\$287.846,00, obtido na Tabela 1. 
Tabela 1 - Obtenção do Investimento Inicial.

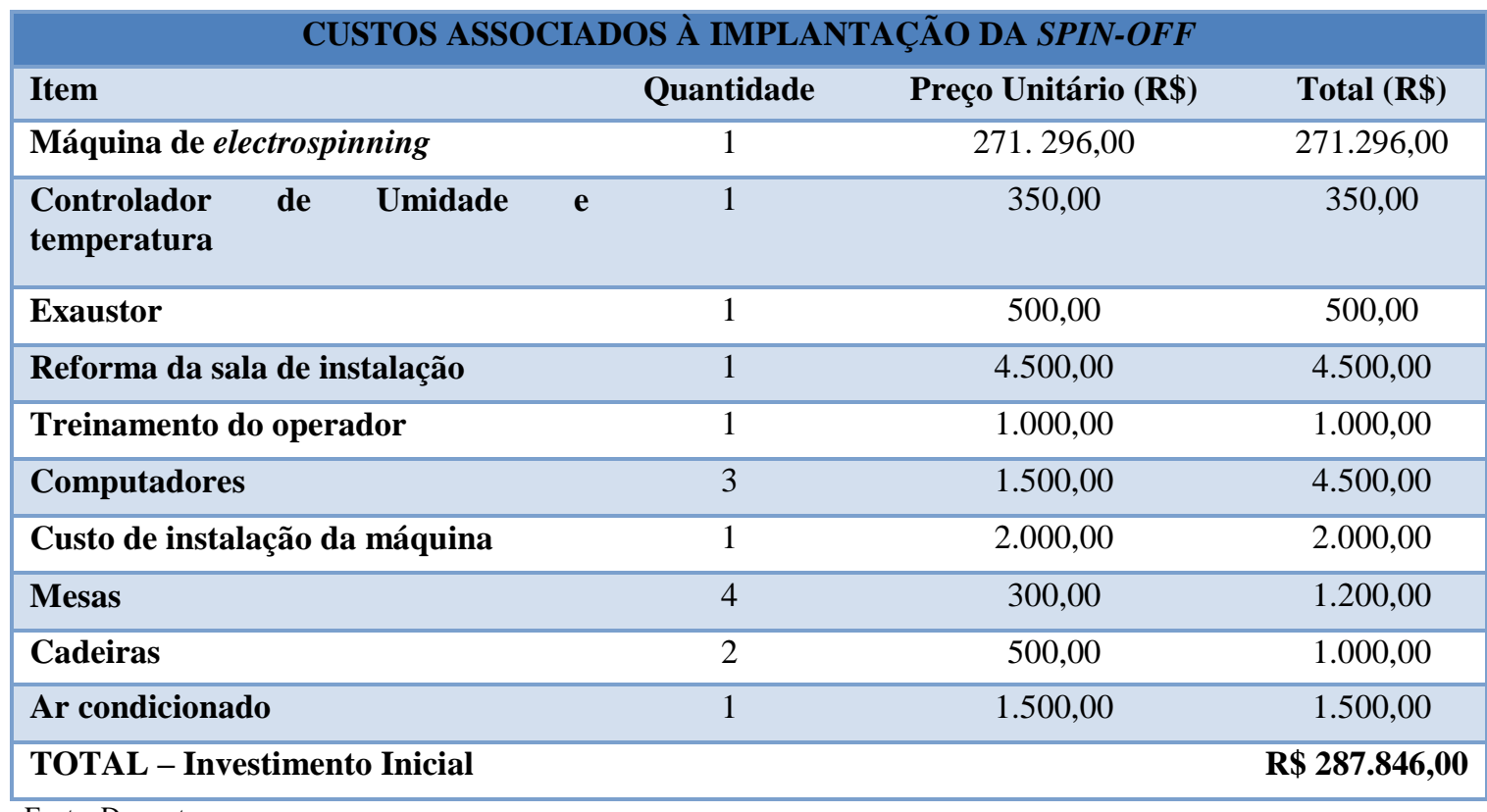

Os custos fixos associados ao projeto são listados abaixo na Tabela 2.

Tabela 2 - Custos fixos da operação.

\begin{tabular}{|lccc|}
\hline Item & CUSTOS FIXOS & & \\
\hline Mão de Obra do Operador (com encargos) & 1,00 & $5.680,00$ & Preço Unitário (R\$) \\
\hline Telefone e Internet & 1,00 & 500,00 & $5.680,00$ \\
\hline Outros (limpeza) & 1,00 & 700,00 & 700,00 \\
\hline Valor mensal & & & $6.880,00$ \\
\hline Total por ano & & & $\mathrm{R} \$ 82.560,00$ \\
\hline
\end{tabular}

Fonte: Dos autores.

Alguns custos variáveis decorrentes da execução do projeto como custos de manutenção do equipamento, matéria prima, energia e gastos com o tratamento dos gases liberados na atmosfera, todos estes listados na Tabela 3.

Tabela 3 - Custos variáveis da operação.

\begin{tabular}{|lccc|}
\hline Item & $\begin{array}{c}\text { CUSTOS VARIÁVEIS } \\
\text { Quant. } \\
\text { mensal }\end{array}$ & $\begin{array}{c}\text { Preço Unitário } \\
(\mathbf{R} \$)\end{array}$ & Total (R\$) / ano \\
\hline Manutenção & 1 & 500 & 6.000 \\
\hline Matéria prima (polímeros e outros insumos) & 20 & $1.000,00$ & $20.000,00$ \\
\hline Energia (kW/h) & 34.000 & 0,2 & $81.600,00$ \\
\hline Tratamento dos resíduos gasosos & 1 & 1000 & $12.000,00$ \\
\hline & & Total por ano & R \$ 119.600,00 \\
\hline
\end{tabular}

Fonte: Dos autores. 
No decorrer de dez anos de uso, o equipamento de electrospinning sofrerá uma depreciação, ficando com valor residual em torno de $20 \%$ do valor de investimento inicial, passado o tempo de funcionamento, conforme apresentado na Tabela 4.

Tabela 4 - Depreciação da máquina.

\begin{tabular}{|lr|}
\hline \multicolumn{2}{|c|}{ DEPRECIAÇÃO } \\
\hline Investimento inicial & $\mathrm{R} \$ 287.846,00$ \\
\hline Valor sucata & $\mathrm{R} \$ 57.569,20$ \\
\hline Vida útil (anos) & 10 \\
\hline Horas ano & 1320 \\
\hline Depreciação/h & $\mathbf{R} \$ \mathbf{1 7 , 4 5}$ \\
\hline
\end{tabular}

Fonte: Dos autores.

Tendo esses dados listados acima, pode-se efetuar uma análise financeira da aquisição do equipamento com relação à viabilidade do mesmos de acordo com as análises financeiras a partir da técnica do valor presente líquido (VPL) e da taxa interna de retorno (TIR).

\subsubsection{Plano Financeiro}

O plano financeiro a ser utilizado na análise econômica do projeto está detalhado na Tabela 5. Os dados desta tabela forma obtidos levando em consideração os cálculos realizados anteriormente para o Investimento Inicial, valor imobilizado após depreciação, assim como considerando uma taxa mínima de atratividade de $8 \%$ e um aumento de custos e preços em 5\%. Os impostos foram obtidos a partir da Tabela Simples Nacional (conforme Anexo 1) de acordo com a previsão de faturamento da spin-off, leva-se em consideração também no plano financeiro que o técnico que irá manipular o equipamento deverá ter uma jornada diária de seis horas nessa atividade.

Tabela 5 - Plano Financeiro.

\begin{tabular}{|lcl|}
\hline \multicolumn{1}{|c|}{ Item } & VLANO FINANCEIRO & \multicolumn{1}{c|}{ Observação } \\
\hline Investimento inicial & $\mathrm{R} \$ 287.846,00$ & Equipamento e instalação \\
\hline TMA & $8 \%$ & Ao ano (aplicação financeira) \\
\hline Aumento de custos & $5 \%$ & Ao ano \\
\hline Aumento de preços & $5 \%$ & Ao ano \\
\hline Impostos & $\begin{array}{c}\text { Tabela Simples } \\
\text { Nacional }\end{array}$ & $\begin{array}{l}\text { Devido à previsão de faturamento da spin- } \\
\text { off }\end{array}$ \\
\hline Horas trabalhadas por turno & $6 \mathrm{~h}$ & Semana com 5 dias úteis \\
\hline Vida útil do equipamento & 10 & Anos \\
\hline $\begin{array}{l}\text { Valor imobilizado após } \\
\text { depreciação (equipamentos) }\end{array}$ & $\mathrm{R} \$ 57.569,20$ & $(20 \%)$ \\
\hline Fonte: Dos autores. & & \\
\hline
\end{tabular}

Tabela 6 - Formação do preço de venda. 


\begin{tabular}{|c|c|c|c|}
\hline Custos diretos e indiretos & Unitário & Quant. & Valor R\$ \\
\hline Polímeros & 5,6 & 0,56 & 3,14 \\
\hline Energia / mês & 4600 & 39.600 & 0,28 \\
\hline Água / mês & 282,36 & 39.600 & 0,01 \\
\hline Tratamento de resíduos /mês & 250 & 39.600 & 0,01 \\
\hline Manutenção /mês & 1800 & 39.600 & 0,05 \\
\hline Custo Total Agregado /mês & 8793,6 & 39.600 & 0,22 \\
\hline Total & \multicolumn{3}{|c|}{3,70} \\
\hline Despesas com vendas & \multicolumn{2}{|c|}{$6 \%$} & 3,92 \\
\hline Lucro & \multicolumn{2}{|c|}{$20 \%$} & 5,02 \\
\hline Impostos (Simples Nacional) & \multicolumn{2}{|c|}{$8,1 \%$} & 5,56 \\
\hline Preço de Venda & \multicolumn{3}{|c|}{7,16} \\
\hline
\end{tabular}

No cálculo do preço de venda são consideradas a premissas de que o mesmo deve englobar todos os custos de produção, sejam eles diretos ou indiretos, os impostos gerados, além de uma margem de contribuição que suporte as despesas do negócio. Adicionalmente, deverá proporcionar o lucro liquido esperado no negócio.

Na Tabela 6 pode ser visto o cálculo de formação do preço de venda das fibras poliméricas.

A produção de fibras, segundo o fabricante, é de 0 a $500 \mathrm{~mm}$ por minuto. Supondo o preço de R\$ 7,16 por metro, trabalho em produção máxima e com o tempo de inicialização de operação da máquina em 30 minutos por jornada de trabalho temos a previsão da receita conforme apresentado na Tabela 7.

Tabela 7 - Receita prevista do projeto.

\begin{tabular}{|ccrr|}
\hline & PREVISÃO DE RECEITA & & \\
\hline & Tempo (h) & $\begin{array}{r}\text { Comprimento } \\
\text { produzido (m) }\end{array}$ & $\begin{array}{r}\text { Preço / } \begin{array}{r}\text { Metro } \\
(\mathrm{R} \$)\end{array} \\
\hline \text { Produção máxima/hora }\end{array}$ \\
\hline Produção anual & 1 & 30 & 7,16 \\
\hline & Total (primeiro ano) & 39.600 & \\
\hline & & & $\mathbf{2 8 3 . 5 3 6 , 0 0}$ \\
\hline
\end{tabular}

De acordo com Vedovello e Figueiredo (2005), nos anos recentes a infraestrutura tecnológica tem despertado crescente interesse em diversos segmentos políticos e socioeconômicos, incluindo os tomadores de decisão, agências de desenvolvimento, empreendedores, bem como as comunidades acadêmicas e de pesquisa. O foco das políticas industriais e de inovação tem, gradualmente, mudado de um uso dominante e/ou exclusivo de instrumentos diretos de apoio para outras formas mais indiretas. Com o objetivo de melhorar o ambiente competitivo das empresas, esforços consideráveis têm sido canalizados para a construção e reforço da infraestrutura tecnológica, em particular na implementação e desenvolvimento de incubadoras de empresas. 
Universidades e centros de pesquisa vêm propondo alternativas interessantes a partir de pesquisas de ponta para este particular tema, mas há ainda alguma dificuldade de se ultrapassar as barreiras acadêmicas e implementar um negócio Alternativas inteligentes visando uma maior interação universidade-empresa tem recebido a denominação de 'spin-off'. De fato, de acordo com Santana e Martins (2009), uma 'spin-off' refere-se a uma nova empresa que nasceu a partir de um grupo de pesquisa de uma empresa, universidade ou centro de pesquisa público ou privado, normalmente com o objetivo de explorar um novo produto ou serviço de alta tecnologia.É comum que estas se estabeleçam em incubadoras de empresas ou áreas de concentração de empresas de alta tecnologia. Os 'spin-offs' podem ser definidos como empreendimentos criados com a intenção de viabilizar a transferência, formal ou informal, de tecnologia e conhecimento gerados em instituições científicas (universidades, centros de pesquisa etc.) através do lançamento de produtos e serviços no mercado. (GOMES e SALERNO, 2010).

Uma das principais vantagens observadas neste projeto integrado de negócios a partir de uma 'spin-off' refere-se ao baixo valor do investimento inicial, frente ao potencial de geração de resultado verificado pela forte tendência de mercado por produtos altamente tecnológicos que empregam nanofios ou nanofibras (NASCIMENTO et al., 2016). Outras vantagens também podem ser visualizadas nos resultados financeiros previstos explicitados e detalhados a seguir. 


\subsection{Resultados financeiros previstos}

Os resultados previstos na análise financeira são detalhados na Tabela 8 .

Tabela 8 - Resultados financeiros previstos.

\begin{tabular}{|c|c|c|c|c|c|c|c|c|c|c|c|c|}
\hline DRE & & & & & & & & & & & & \\
\hline & & & & & & & & & & & & \\
\hline \multirow[t]{2}{*}{ RECEITAS } & & ANOS & & & & & & & & & & \\
\hline & & 1 & 2 & 3 & 4 & 5 & 6 & 7 & 8 & 9 & 10 & TOTAL \\
\hline Preço / m & & 7,16 & 7,52 & 7,89 & 8,29 & 8,70 & 9,14 & 9,60 & 10,07 & 10,58 & 11,11 & \\
\hline Produção (m) & & $39.600,00$ & $39.600,00$ & $39.600,00$ & $39.600,00$ & $39.600,00$ & $39.600,00$ & $39.600,00$ & $39.600,00$ & $39.600,00$ & $39.600,00$ & \\
\hline TOTAL RECEITAS BRUTAS & & $283.536,00$ & $297.712,80$ & $312.598,44$ & 328.228,36 & $344.639,78$ & 361.871,77 & $379.965,36$ & $398.963,63$ & 418.911,81 & $439.857,40$ & $3.566 .285,34$ \\
\hline Simples Nacional (Anexo 1) & & $16.927,10$ & $17.773,45$ & $18.662,13$ & $19.595,23$ & $20.574,99$ & $26.561,39$ & $27.889,46$ & $29.283,93$ & $30.748,13$ & $32.285,53$ & $240.301,34$ \\
\hline Despesas & & & & & & & & & & & & 0,00 \\
\hline Custos Fixos & & $82.560,00$ & $82.560,00$ & $82.560,00$ & $82.560,00$ & $82.560,00$ & $82.560,00$ & $82.560,00$ & $82.560,00$ & $82.560,00$ & $82.560,00$ & $825.600,00$ \\
\hline \multicolumn{13}{|c|}{ 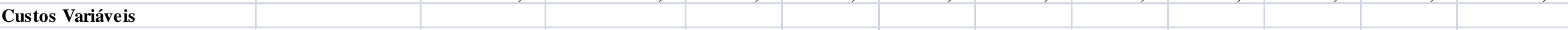 } \\
\hline Manutenção & & $6.000,00$ & $6.300,00$ & $6.615,00$ & $6.945,75$ & $7.293,04$ & $7.657,69$ & $8.040,57$ & $8.442,60$ & $8.864,73$ & $9.307,97$ & $75.467,36$ \\
\hline Materia-prima & & $20.000,00$ & $21.000,00$ & $22.050,00$ & $23.152,50$ & $24.310,13$ & $25.525,63$ & $26.801,91$ & $28.142,01$ & $29.549,11$ & $31.026,56$ & $251.557,85$ \\
\hline Energia $(\mathrm{kW} / \mathrm{h})$ & & $81.600,00$ & $85.680,00$ & $89.964,00$ & $94.462,20$ & $99.185,31$ & $104.144,58$ & $109.351,80$ & $114.819,39$ & $120.560,36$ & $126.588,38$ & $1.026 .356,03$ \\
\hline Tratamento dos resíduos gasosos & & $12.000,00$ & $12.600,00$ & $13.230,00$ & $13.891,50$ & $14.586,08$ & $15.315,38$ & $16.081,15$ & $16.885,21$ & $17.729,47$ & $18.615,94$ & $150.934,71$ \\
\hline Total Custos Variáveis & & $119.600,00$ & $125.580,00$ & $131.859,00$ & $138.451,95$ & $145.374,55$ & $152.643,27$ & $160.275,44$ & $168.289,21$ & $176.703,67$ & $185.538,85$ & $1.504 .315,95$ \\
\hline Investimento & $287.846,00$ & & & & & & & & & & & \\
\hline LUCRO LÍQUIDO & $-287.846,00$ & $64.448,90$ & $71.799,35$ & $79.517,31$ & $87.621,18$ & $96.130,24$ & $100.107,11$ & $109.240,46$ & $118.830,48$ & $128.900,01$ & $139.473,01$ & $996.068,05$ \\
\hline VPL & $346.448,21$ & & & & & & & & & & & \\
\hline TIR & $27 \%$ & & Simples Nacional & $5,97 \%$ & & & & & & & & \\
\hline TMA & $8 \%$ & & & $7,34 \%$ & & & & & & & & \\
\hline Payback (anos) & 4,466267018 & & & & & & & & & & & \\
\hline
\end{tabular}

Fonte: Dos autores. 


\section{Conclusões}

O processo de desenvolvimento de produtos em spin-offs acadêmicos ainda é um tópico pouco explorado na literatura. Apesar da importância desse processo para esse tipo de firma e do impacto que os produtos gerados a partir de pesquisas científicas têm na sociedade, o número de estudos que investigam o processo, as suas características e as suas etapas ainda é pequeno, e pouco relacionado a empresas de alta tecnologia, como as que empregam nanofibras e nanofios.

Este artigo enfoca a transferência de conhecimento da infraestrutura tecnológica para a indústria, a partir da experiência de um laboratório de produção de nanofios brasileiro que pode servir como uma incubadora de inovação.

A partir dos resultados da análise de viabilidade econômica, pode-se verificar que o retorno obtido pelo investimento torna o projeto viável, de acordo com as duas técnicas utilizadas (VPL e TIR), onde foi obtida uma taxa interna de retorno de $27 \%$ e um valor presente líquido de $\mathrm{R} \$$ 346.448,21. Além dessas vantagens financeiras, tem-se a oportunidade de, a partir do funcionamento da empresa, lançar no mercado novos materiais com propriedades únicas e inovadoras, características que somente a utilização da nanotecnologia pode oferecer.

Assim, como contribuição para a prática, espera-se que este modelo subsidie pesquisadores e empresários no processo de incorporação de tecnologia a produtos e no planejamento de negócios para outras empresas de natureza similar. Espera-se ainda que o modelo possa ajudar incubadoras e investidores na condução de ações que auxiliem no desenvolvimento e na promoção de spin-offs acadêmicos.

\section{Abstract}

Production nanofibres from the electrospinning technique is a national strategic line of actuation due to the variety of materials which can be obtained as well as a wide range of applications. Polymer nanocomposites, generally constituted by the union of polymer with an inorganic substances, makes possible to obtain new materials with unique and optimized characteristics when compared with isolated materials. The electrospinning technique causes a much more effective interaction between the two materials in the fiber formation. Aiming the better use of this technique, this project represents a financially analysis of the feasibility of purchasing and installing an academic spin-off to the fabrication of nanowires by electrospinning for a high tech incubator of innovation linked to a Brazilian materials research laboratory. From the use of the economic analysis methods of Net Present Value and Internal Rate of Return was possible to verify the potential profitability of the acquisition and operation of an electrospinning equipment that can provide for a spin-off, considering both the initial investment necessary as the peculiarities and variations presented by the market in which the product is inserted. 
Key-words: polymer fiber; electrospinning; spin-off; economic viability

\section{Referências}

ALVES, A.K., Andrade, P.A., Berutti, F.A. Bergmann C.P. Caracterização de nanofibras de zircônia obtidas por electrospinning. Anais do $\mathbf{1 7}^{\mathbf{0}}$ Congresso Brasileiro de Engenharia e Ciência dos Materiais, 2006, 1414-1422.

ARAUJO, E. S. ; NASCIMENTO, M. L. F. ; DE OLIVEIRA, H. P. Influence of Triton X-100 on PVA Fibres Production by the Electrospinning Technique. Fibres \& Textiles in Eastern Europe, v. 21, p. 39-43, 2013.

ARAUJO, E. S. , NASCIMENTO, M. L. F. , de OLIVEIRA, H. P. Electrospinning of Polymeric Fibres: An Unconventional View on the Influence of Surface Tension on Fibre Diameter. Fibres \& Textiles in Eastern Europe, v. 24, p. 22-29, 2016. crossref

CONG, Y., LIU, S., CHEN, H. Fabrication of Conductive Polypyrrole Nanofibers by Electrospinning. Journal of Nanomaterials, v. 2013, 1-6, 2013. crossref

COSTA, F. F. P., ARAUJO, E. S. , NASCIMENTO, M. L. F. , DE OLIVEIRA, H. P. Electrospun Fibers of Enteric Polymer for Controlled Drug Delivery. International Journal of Polymer Science, v. 2015, p. 1-8, 2015. cross ref

COSTA, R. G. F., OLIVEIRA, J. E. PAUlA, G. F. PICCIANI, P. H. S. MEDEIROS, E. S. RIBEIRO, C. MATTOSO

L. H. C. Eletrofiação de polímeros em solução: parte II: aplicações e perspectivas. Polímeros [online].vol.22, n.2, pp. 178-185. Epub Mar 13, 2012. crossref

DIAS, A. A., PORTO, G. S. Gestão de transferência de tecnologia na inova Unicamp. Rev. Adm. Contemp. [online] vol.17, 2013

ELMARCO NS LAB. Product profile. Nanospider ${ }^{\text {TM }}$ Lab products. Disponível em: http://www.elmarco.com. Acesso em : 21 de maio de 2016.

FORMHALS, A. Process and Apparatus for Preparing Artificial Threads. US Patent 1,975,504 (1934).

FRENOT, A., CHRONAKIS, I.S. Polymer nanofibers assembled by electrospinning. Current opinion in Colloid and interface science, v. 8, 64-75, 2003. crossref

GITMAN, L. Princípios de administração financeira. 12ª ed. São Paulo: Pearson,. 2010

GOMES A. V. L., SALERNO, S. M. Modelo que integra processo de desenvolvimento de produto e planejamento inicial de spin-offs acadêmicos. Gestão \&. Produção. São Carlos, v. 17, n. 2, p. 245-255, 2010.

GUERRINI, L. M., BRANCIFORTI, M. C., BRETAS, R. E. S., OLIVEIRA M. P.. Eletrofiação do Poli(álcool vinílico) via solução aquosa. Polímeros: Ciência e Tecnologia, v. 16, 286-293, 2006. crossref

LAGO, R.M.; ARAÚJO, M.H.; OLIVEIRA, L.C.A.; CABRAL, P.R.M.; CHENG, L.C.; BORGES, C.; FILION, L.J. Spin-off acadêmico: criando riquezas a partir de conhecimento e pesquisa. Química Nova, vol. 28, S26-35, 2005. crossref

NASCIMENTO, M. L. F., ARAUjO, E. S. , CORDEIRO, E. R. , OLIVEIRA, A. H. P. , DE OLIVEIRA, H. P. A Literature Investigation about Electrospinning and Nanofibers: Historical Trends, Current Status and Future Challenges. Recent Patents on Nanotechnology, v. 9, 76-85, 2015. crossref

OLIVEIRA, A. H. P. Nanocompósito polímero/semicondutor e polímero/nanotubo de carbono: aplicação em sensores de umidade e fotocatalizadores. Dissertação (Mestrado em Ciência dos Materiais) - Universidade Federal do Vale do São Francisco, 2012.

PORTELA, P.M.D. Montagem e Automatização de um Sistema de Electrospinning. Universidade do Minho , 2010 , 7-38.

SAMANEZ, Carlos Patrício. Engenharia econômica. São Paulo: Pearson Prentice Hall, 2009.

SANTANA, J.R., MARTINS, F.A. Interagir para inovar. Cartilha Relação Universidade-Empresa. UFS, 2009. 
TABOSA, et. al. Análise de viabilidade econômico-financeira de um empreendimento imobiliário. Anais do XXXII Encontro Nacional de Engenharia de Produção - ENEGEP. 2012.

VEDOVEllo, C., FIGUEIREDO, P.N. RAE-eletrônica, $\quad$ v. $\quad 4, \quad$ n. $\quad 1$, Art. $\quad 10, \quad$ jan./jul. 2005 http://www.rae.com.br/eletronica/index.cfm. crossref 


\section{Anexo 1}

Tabela Simples Nacional

\section{Partilha do Simples Nacional - Indústria}

(Redaçäo dada pela Lei Complementar n 139, de 2011 - produção de efeitos a partir de $1^{\circ}$ de janeiro de 2012).

\begin{tabular}{|c|c|c|c|c|c|c|c|c|}
\hline $\begin{array}{l}\text { Receita Bruta em } 12 \text { meses (em } \\
\text { R\$) }\end{array}$ & LÍQUOTA & IRPJ & CSLL & COFINS & PIS/PASEP & CPP & ICMS & IPI \\
\hline Até $180.000,00$ & $4,50 \%$ & $0,00 \%$ & $0,00 \%$ & $0,00 \%$ & 0,0070 & $2,75 \%$ & $1,25 \%$ & $0,50 \%$ \\
\hline De $180.000,01$ a $360.000,00$ & $5,97 \%$ & $0,00 \%$ & $0,00 \%$ & $0,86 \%$ & $0,00 \%$ & $2,75 \%$ & $1,86 \%$ & $0,50 \%$ \\
\hline De $360.000,01$ a $540.000,00$ & $7,34 \%$ & $0,27 \%$ & $0,31 \%$ & $0,95 \%$ & $0,23 \%$ & $2,75 \%$ & $2,33 \%$ & $0,50 \%$ \\
\hline De $540.000,01$ a $720.000,00$ & $8,04 \%$ & $0,35 \%$ & $0,35 \%$ & $1,04 \%$ & $0,25 \%$ & $2,99 \%$ & $2,56 \%$ & $0,50 \%$ \\
\hline De $720.000,01$ a $900.000,00$ & $8,10 \%$ & $0,35 \%$ & $0,35 \%$ & $1,05 \%$ & $0,25 \%$ & $3,02 \%$ & $2,58 \%$ & $0,50 \%$ \\
\hline De $900.000,01$ a $1.080 .000,00$ & $8,78 \%$ & $0,38 \%$ & $0,38 \%$ & $1,15 \%$ & $0,27 \%$ & $3,28 \%$ & $2,82 \%$ & $0,50 \%$ \\
\hline De $1.080 .000,01$ a $1.260 .000,00$ & $8,86 \%$ & $0,39 \%$ & $0,39 \%$ & $1,16 \%$ & $0,28 \%$ & $3,30 \%$ & $2,84 \%$ & $0,50 \%$ \\
\hline De $1.260 .000,01$ a $1.440 .000,00$ & $805 \%$ & $0,39 \%$ & $0,39 \%$ & $1,17 \%$ & $0,28 \%$ & $3,35 \%$ & $2,87 \%$ & $0,50 \%$ \\
\hline De $1.440 .000,01$ a $1.620 .000,00$ & $053 \%$ & $0,42 \%$ & $0,42 \%$ & $1,25 \%$ & $030 \%$ & $3,57 \%$ & $3,07 \%$ & $0,50 \%$ \\
\hline De $1.620 .000,01$ a $1.800 .000,00$ & $060 \%$ & $0,42 \%$ & $0,42 \%$ & $1,26 \%$ & $0,30 \%$ & $3,62 \%$ & $3,10 \%$ & $0,50 \%$ \\
\hline De $1.800 .000,01$ a $1.980 .000,00$ & 10.4 & $46 \%$ & $0,46 \%$ & 138 & 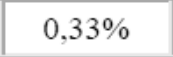 & $4 \%$ & $3,38 \%$ & $0,50 \%$ \\
\hline De $1.980 .000,01$ a $2.160 .000,00$ & $10,54 \%$ & $0,46 \%$ & $0,46 \%$ & $1,39 \%$ & 0,3 & $3,99 \%$ & $3,41 \%$ & $0,50 \%$ \\
\hline De $2.160 .000,01$ a $2.340 .000,00$ & $10,63 \%$ & $0,47 \%$ & $47 \%$ & $1,40 \%$ & 0,3 & $4,01 \%$ & $3,45 \%$ & $0,50 \%$ \\
\hline De $2.340 .000,01$ a $2.520 .000,00$ & 10,7070 & $0,47 \%$ & $0,47 \%$ & 1,4270 & $0,54 \%$ & $4,05 \%$ & $3,48 \%$ & $0,50 \%$ \\
\hline De $2.520 .000,01$ a $2.700 .000,00$ & $10,82 \%$ & $0,48 \%$ & $0,48 \%$ & $1,+570$ & 0,3470 & $4,08 \%$ & $3,51 \%$ & $0,50 \%$ \\
\hline De $2.700 .000,01$ a $2.880 .000,00$ & $11,73 \%$ & $0,52 \%$ & $0,52 \%$ & $1,56 \%$ & $0,5 / \%$ & $4,44 \%$ & $3,82 \%$ & $0,50 \%$ \\
\hline De $2.880 .000,01$ a $3.060 .000,00$ & $11,82 \%$ & $0,52 \%$ & $0,52 \%$ & $1,57 \%$ & $0,5 / \%$ & $4,49 \%$ & $3,85 \%$ & $0,50 \%$ \\
\hline De $3.060 .000,01$ a $3.240 .000,00$ & $11,92 \%$ & $0,53 \%$ & $0,53 \%$ & $1,58 \%$ & $0,38 \%$ & $4,52 \%$ & $3,88 \%$ & $0,50 \%$ \\
\hline De $3.240 .000,01$ a $3.420 .000,00$ & $12,01 \%$ & $0,53 \%$ & $0,53 \%$ & $1,60 \%$ & $0,38 \%$ & $4,56 \%$ & $3,91 \%$ & $0,50 \%$ \\
\hline De $3.420 .000,01$ a $3.600 .000,00$ & $12,11 \%$ & $0,54 \%$ & $0,54 \%$ & $1,60 \%$ & $0,38 \%$ & $4,60 \%$ & $3,95 \%$ & $0,50 \%$ \\
\hline
\end{tabular}

Fonte: Receita Federal do Brasil 


\section{Dados dos autores}

Nome completo: Ariadne Helena Pequeno de Oliveira

Filiação institucional: Universidade Federal da Bahia

Departamento: Departamento de Engenharia Química

Função ou cargo ocupado: Doutoranda em Engenharia Industrial

Endereço completo para correspondência: LEIMO/IPCM - Instituto de Pesquisa em Ciência dos Materiais, Av. Antonio Carlos Magalhães 510, Country Club. Juazeiro, BA, Brasil, CEP 48902-300

Telefones para contato: (71) 3283-9803

E-mail: ariadne.helena@ufba.br

Nome completo: Luciano Pisanu

Filiação institucional: Universidade Federal da Bahia

Departamento: Departamento de Engenharia Química

Função ou cargo ocupado: Doutorando em Engenharia Industrial

Endereço completo para correspondência: SENAI Cimatec, Av. Orlando Gomes 1845, Piatã.

Salvador, BA, Brasil, CEP 41650-010

Telefones para contato: (71) 3462-8431

E-mail: luciano.pisanu@ fieb.org.br

Nome completo: Helinando Pequeno de Oliveira

Filiação institucional: Universidade Federal do Vale do São Francisco

Departamento: Programa de Pós-Graduação em Ciência dos Materiais

Função ou cargo ocupado: Professor

Endereço completo para correspondência: LEIMO/IPCM - Instituto de Pesquisa em Ciência dos

Materiais, Av. Antonio Carlos Magalhães 510, Country Club. Juazeiro, BA, Brasil, CEP 48902-300

Telefones para contato: (74) 2102-7645

E-mail: helinando.oliveira@ univasf.edu.br

Nome completo: Marcio Luis Ferreira Nascimento

Filiação institucional: Universidade Federal da Bahia

Departamento: Departamento de Engenharia Química

Função ou cargo ocupado: Professor

Endereço completo para correspondência: PROTEC / PEI - Programa de Pós-Graduação em Engenharia Industrial, Rua Professor Aristides Novis 2, Federação. Salvador, BA, Brasil, CEP 40210-630

Telefones para contato: (71) 3283-9803

E-mail:mlfn@ufba.br

Submetido em: 21-04-2016

Aceito em: 21-09-2016 